\title{
PEUT-ON SE PASSER DE LA VOITURE HORS DES CENTRES URBAINS ?
}

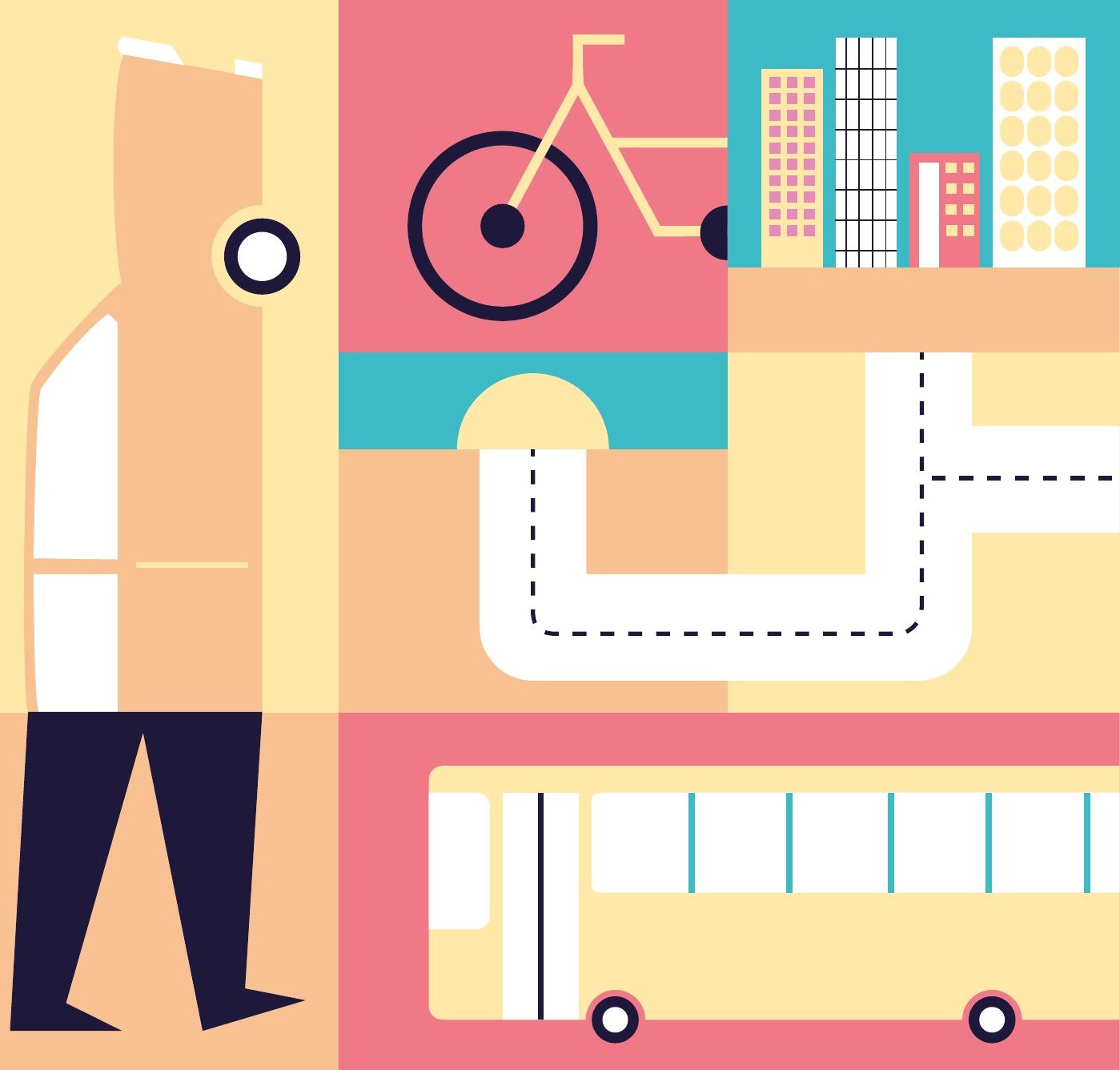

DIRECTION

Yoann Demoli
COLLOQUE

27 février 2020

UVSQ - Laboratoire Printemps Guyancourt 


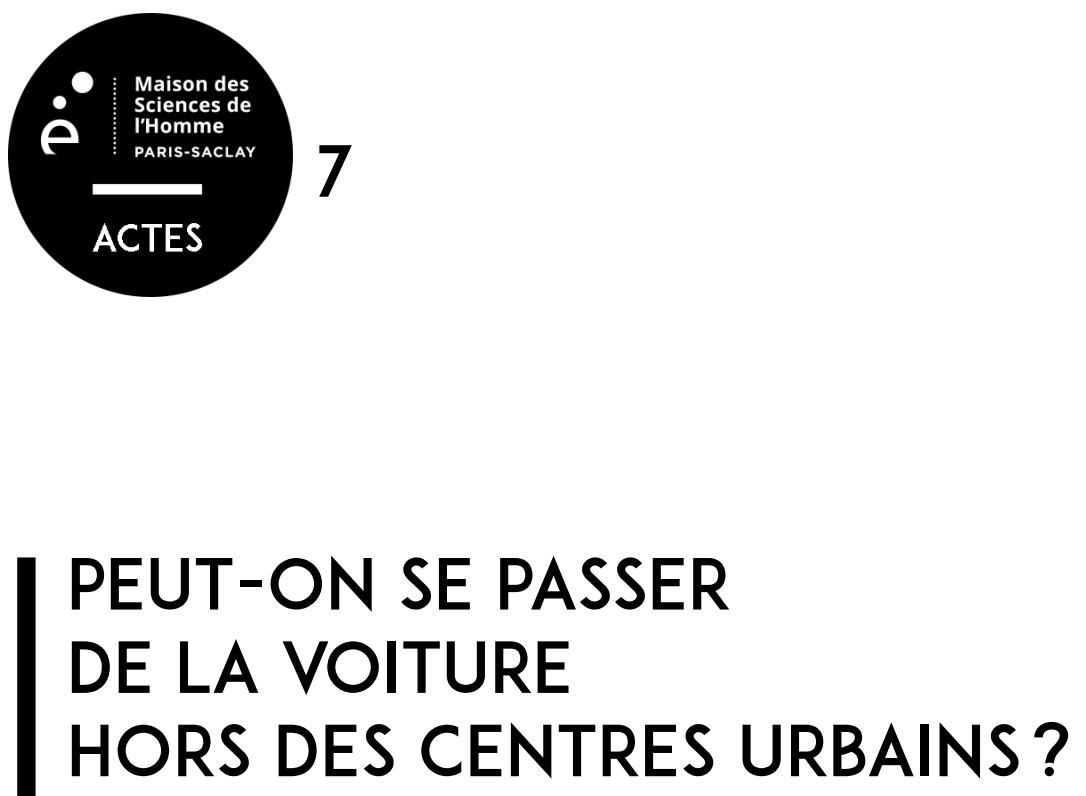

\section{COLLOQUE}

27 février 2020

UVSQ - Laboratoire Printemps

Guyancourt

\section{DIRECTION}

Yoann Demoli 


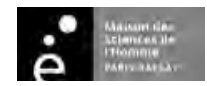

(c)MSH Paris-Saclay Éditions, 2021.

4, avenue des Sciences, 91190 Gif-sur-Yvette www.msh-paris-saclay.fr

ISBN 978-2-490369-06-5 


\section{Tiers-lieux et espaces de coworking à la campagne : quel impact sur les mobilités ?}

Aurore Flipo

Au cours des cinq dernières années, on a pu assister à la remise du télétravail sur l'agenda des politiques publiques, à la faveur de l'apparition et du développement des espaces de coworking (EC) et autres « tierslieux " (Scaillerez \& Tremblay, 2017). La promesse d'une meilleure qualité de vie et de meilleures conditions de travail, mais aussi d'une plus grande durabilité économique et écologique, est ainsi portée par ces espaces. Le soutien des pouvoirs publics au développement des EC et des tierslieux repose notamment sur des effets présumés vertueux, mais encore non démontrés, du coworking en matière de mobilité durable (Lejoux et al., 2019). En offrant aux actifs la possibilité de travailler à proximité de leur domicile, les EC sont supposés permettre une réduction des mobilités domicile-travail et favoriser les mobilités douces. L'ADEME', par exemple, identifie dans le cadre de son scénario Alléger l'empreinte environnementale de la consommation des Français en 2030, les tiers-lieux comme des «solutions d'immobilités ", à même de favoriser une mobilité à " empreinte environnementale allégée " (ADEME, 2014 : 24).

Phénomène urbain à l'origine, les données récentes montrent que ce sont les tiers-lieux situés hors agglomération qui ont crû le plus fortement entre 2016 et 2018 (Levy-Waitz, 2018). Ils représentent $42 \%$ de l'ensemble des espaces français et jusqu'à $70 \%$ dans certaines régions, dans lesquelles des politiques publiques volontaristes ont été mises en

Agence De l'Environnement et de la Maîtrise de l'Énergie. 
place (Nouvelle-Aquitaine, Occitanie). Pour celles-ci, les tiers-lieux ont vocation à structurer le territoire et les mobilités : il s'agit de réduire les déplacements liés à l'emploi mais aussi aux services, en assumant une multiplicité de fonctions de "mobilité inversée " ( $\mathrm{MSAP}^{2}$, relais postal, groupement d'achats, université à distance, etc.).

Dans cet article, nous souhaitons présenter les premiers résultats d'une enquête menée dans le cadre de l'ANR Coworkworlds ${ }^{3}$ (2017-2020) dans la région Auvergne-Rhône-Alpes. À travers l'examen de l'historique de ces lieux, des trajectoires des individus qui les fréquentent, ainsi que de leurs habitudes de mobilité quotidienne, nous tenterons d'appréhender la question d'un rôle des tiers-lieux sur la mobilité, ainsi que ses éventuels effets bénéfiques sur les altermobilités ${ }^{4}$, qui dépendent de conditions bien précises qu'il s'agira d'étayer.

\section{Coworking, durabilité et ruralité : état des connaissances}

\section{Le coworking, un objet nouveau et de plus en plus diffus}

Apparus en Californie en 2006, les EC se sont progressivement diffusés dans le monde à partir de 2010 autour de nouveaux besoins de travailleurs « qui n'ont plus besoin de lieu fixe pour travailler, mais d'une communauté de travail ${ }^{5}$ ». Ceux-ci émergent à la faveur du développement des technologies de l'information et de la communication, du numérique, de la conception, et plus largement de l'ensemble des professions

2 Maison de Service Au Public.

3 Projet de l'Agence Nationale de la Recherche ANR-17-CE22-0004 sur les durabilités et spatialités des pratiques de mobilité des coworkers.

4 Définies comme les alternatives à l'autosolisme et plus largement à la voiture individuelle.

5 Cf. le Workshop "Les espaces de coworking. Nouveaux lieux ? Nouveaux liens ? Nouvelle économie ? ", organisé par Christine Liefooghe, Christian Mahieu et Michel David, à la MESHS, à Lille, le 19 mars 2013, dans le cadre du séminaire POPSU (Plate-forme d'Observation des Projets et Stratégies Urbaines). Voici l'URL de présentation du Workshop : http://anis.asso.fr/IMG/pdf/coworkingpopsu19mars13-preprogramme.pdf (consulté le 29/09/2018). 
parfois qualifiées de " créatives " (Gill \& Pratt, 2008 ; Gandini, 2015 ; Spinuzzi, 2012 ; entre autres).

Le terme de tiers-lieu désigne une galaxie plus vaste d'espaces (Flipo \& Lejoux, 2020). Il est emprunté à Ray Oldenburg (1999 [1989]) et s'entend par opposition au domicile (premier lieu) et à l'entreprise (second lieu). Les tiers-lieux sont définis par Oldenburg comme des lieux ouverts, propices à la sociabilité et de mixité (Burret, 2017). Lapparition des EC résulterait d'une spécialisation des tiers-lieux en diverses fonctionnalités (culturels, éducatifs, de travail, etc.), affirment Patrick Genoud et Alexis Moeckli (2010). Les EC et tiers-lieux de travail se caractérisent donc a minima par une utilisation tarifée d'une part, et par la mise à disposition d'outils matériels d'autre part (Boboc et al., 2014 ; Burret, 2017), mais également par la constitution d'un réseau social et professionnel. La notion de communauté est ainsi centrale dans l'ensemble des définitions du coworking.

\section{Le développement récent des tiers-lieux en milieu rural}

Originellement associés aux hypercentres urbains, ces nouveaux lieux ont fait leur apparition, au cours des cinq dernières années, hors des villes et dans des zones faiblement métropolisées (Leducq, Demazière \& Coquel, 2019), où ils sont souvent multifonctionnels (Besson, 2018). Dans la grande majorité des cas, ces lieux ont été créés avec le soutien, voire à l'initiative, des pouvoirs publics locaux qui travaillent à la mise en relation des acteurs du territoire (Torre, 2018).

Par ailleurs, les pouvoirs publics locaux voient dans le numérique de nombreuses opportunités : développer l'entreprenariat rural pour maintenir l'emploi sur place, mais aussi encourager le télétravail dans le but d'attirer de nouvelles populations. Dès les années 1990, l'État ambitionne de faire du télétravail un outil d'aménagement des territoires ruraux, mis en avant par trois appels d'offres de la DATAR ${ }^{7}$ en 1990, 1992 et 1993.

Eu égard à l'échec relatif des télécentres, nombreux sont les observateurs qui avaient prédit peu de succès au développement du télétravail

\section{Ibid.}

7 Délégation interministérielle à l'Aménagement du Territoire et à l'Attractivité Régionale. 
en milieu rural : du " télémythe » (Claisse, 1997) à l'« image repoussoir » du télétravailleur isolé (Moriset, 2004), il semblait cristalliser toutes les impasses de l'utopie du tout-numérique.

Cependant, les évolutions récentes de la démographie des campagnes, et en particulier des campagnes attractives et résidentielles, témoignent de l'importance des flux des actifs venant des villes vers les campagnes (Pistre, 2012). Investie par des familles avec enfants en bas âge, la possibilité de télétravailler à la campagne, que ce soit en tant que salarié ou comme travailleur indépendant, peut être une solution pour gérer l'augmentation des temps de transports (Ortar, 2009). Si cette pratique était encore il y a une dizaine d'années marginale, elle semble avoir connu une augmentation importante au cours des dernières années (Salgueiro, 2015 ; Flipo, 2020).

\section{Télétravail et espaces de coworking : une assimilation à interroger}

Si la réduction des mobilités domicile-travail par le truchement du télétravail est au cœur de l'argumentaire promouvant les EC et tiers-lieux, cette hypothèse n'a pourtant que peu d'assise empirique. En effet, les études prospectives s'appuient souvent sur les résultats des études sur le télétravail à domicile pour construire des modèles prédictifs (Caisse des Dépôts, 2014 ; CGET, 2015 ; Tinetti et al., 2015 ; Levy-Waitz, 2018). Or, la majorité des coworkers ne sont pas des télétravailleurs salariés à distance et, à l'inverse, la majorité du télétravail ne s'effectue pas dans les EC. Par ailleurs, une partie de ces télétravailleurs n'a pas quitté les locaux de l'employeur pour se rendre dans un EC, mais à leur domicile propre (Spinuzzi, 2012).

Les effets du télétravail sur la mobilité individuelle prise dans son ensemble demeurent en outre ambigus (Aguilera $e t$ al., 20168). Une réduction des trajets contraints grâce au choix du lieu de travail à proximité

8 Cf. également la communication de Elini Kitrinou, "The Impact of Teleworking on Regional Development: A Review ", lors de la $\sigma^{\text {th }}$ International Conference on Applied Financial Economics, qui s'est déroulée à Samos, en Grèce, du 2 au 4 juillet 2009. Les actes sont disponibles en ligne ici : https://www.researchgate. net/publication/235708156_THE_IMPACT_OF_TELEWORKING_ON_ REGIONAL_DEVELOPMENT_A_REVIEW (site consulté le 27/11/2020). 
du domicile est susceptible de générer un effet rebond via l'augmentation des trajets choisis (Bentayou et al., 2019). En outre, il n'est pas évident que les gains observés dans le Grand Paris (Caisse des Dépôts, 2014) ou dans d'autres milieux urbains soient transférables au contexte périurbain et rural. Tissandier et Mariani-Rousset (2019) estiment ainsi que le télétravail provoque une augmentation générale de la mobilité, mais également un report modal vers la marche et les transports en commun en raison de la diminution des distances. Or, en territoire peu dense, les distances peuvent demeurer plus importantes, et l'absence de transports en commun, couplée à la facilité à se déplacer en voiture, peut ne pas inciter au report modal.

Enfin, en permettant de travailler à plus grande distance des villes, les EC en milieu périurbain et rural sont susceptibles de relocaliser des travailleurs dans des zones plus dépendantes de la voiture (Moeckel, 2017). Or, les cadres étant à la fois les populations les plus mobiles - en particulier à l'international et dans le domaine du loisir (Recchi, Flipo \& Duwez, à paraître en mars 2021) -, les plus exposées au télétravail et les moins éloignées de leur emploi (car résidant plus souvent au centre des villes), il n'est pas évident que les gains effectués sur leurs déplacements domicile-travail soient les plus déterminants pour la durabilité des territoires. En revanche, le télétravail peut leur faciliter l'accès aux espaces éloignés, en particulier ruraux (Ortar, 2009). Ces mutations s'observent dans l'augmentation importante du nombre de cadres au cours des dernières années dans les villages isolés (Charmes, 2019).

\section{Méthodologie}

Cette contribution se base sur une enquête réalisée entre 2017 et 2020 dans la région Auvergne-Rhône-Alpes dans le cadre de l'ANR Coworkworlds. Dans un premier temps, un recensement de l'ensemble des EC en fonctionnement au moment de l'enquête a montré que près d'un quart d'entre eux se situaient en territoire peu dense. Dans un second temps, une vingtaine d'espaces ont été visités, essentiellement dans les départements de la Drôme et de l'Ardèche, qui disposent tous deux d'un réseau d'EC dynamique et important comparativement aux autres départements. Des entretiens approfondis ont été effectués avec six fondateurs d'EC et avec une vingtaine de coworkers. De manière complémentaire, une enquête 
quantitative a été administrée auprès de 377 coworkers, dont 59 situés en milieu rural. Enfin, ces différentes sources de données ont été complétées par une enquête ethnographique de deux ans dans un tiers-lieu situé dans une petite ville de la Drôme.

\section{Résultats}

\section{Les profils des coworkers et leurs motivations}

Conformément à ce qui a été relevé ailleurs dans la littérature, la population des coworkers se distingue par un niveau de diplôme très élevé : les deux tiers ont un diplôme de master ou plus, et $94 \%$ de ceux situés en milieu rural sont titulaires d'un diplôme du supérieur. À ce titre, les coworkers ruraux ne diffèrent aucunement de leurs homologues des centres urbains.

Sortir de chez soi est la motivation principale qui a présidé à la fondation de l'EC ou au fait d'en rejoindre un. Pour la plupart des coworkers, c'est la possibilité de faire des rencontres, d'avoir une vie sociale au travail et d'effectuer une distinction entre vie personnelle et vie professionnelle qui a motivé leur choix par rapport au domicile. L'adoption du travail en EC ne leur a pas permis de se rapprocher de leur domicile, mais plutôt de quitter un environnement à la fois professionnel et résidentiel qui ne leur convenait plus, parfois au prix d'une reconversion (Flipo \& Ortar, 2020).

L'enquête révèle que le public des EC ruraux est composé pour moitié d'entrepreneurs (46\%) et pour moitié de salariés (53\%). Ces derniers sont le plus souvent salariés de très petites entreprises et associations domiciliées sur place (27\%), mais on trouve également des salariés d'entreprises plus importantes (25\%), plus souvent situées à distance. Ainsi, les salariés en télétravail à distance sont la plupart du temps très éloignés de leur entreprise : celle-ci n'est pas forcément située dans les villes les plus proches (Lyon et Grenoble), mais peut être située à Paris ou à l'étranger (Berlin, Bruxelles, San Francisco...), voire ne pas avoir de siège physique. Enfin, la proximité de la Drôme et de l'Ardèche avec l'axe de TGV Paris-Marseille permet des retours réguliers dans les grandes villes, que ce soit pour se rendre au siège de leur entreprise ou pour rencontrer des clients. Ainsi, $13 \%$ des coworkers conservent un autre lieu de travail 
fixe qui n'est pas leur domicile propre. La conservation d'une importante mobilité à longue distance explique l'attractivité de la Vallée du Rhône pour les coworkers. Comme l'explique Stéphane, fondateur d'un EC dans la Drôme et consultant en informatique :

Là, je sais qu'on est à quarante minutes de la gare TGV. Si je veux aller en déplacement... Voilà, ça ne m'arrive pas non plus tous les jours mais... Moi le but c'est de développer l'activité là, autour de Drôme-Ardèche, après si j'élargis à Rhône-Alpes s'il y a des missions intéressantes... J'ai toujours des contacts sur Lyon ou sur Paris où je vais de temps en temps... Mais le but c'est surtout de développer une activité ici.

Ainsi, l'un des résultats les plus saillants que l'enquête qualitative a pu faire apparaître est l'importance parmi les coworkers de nouveaux résidents venus des grandes villes, et en particulier de Paris (Flipo, 2020). Certains sont arrivés avec une activité conservée à distance, quand d'autres se sont installés comme indépendants, parfois en conservant le même donneur d'ordres.

\section{La proximité : une distance choisie}

Rares sont les environnements ruraux qui disposent de plus d'un EC dans un même bassin de vie et proposent donc un réel choix, mais quand c'est le cas, on observe que les coworkers ne choisissent pas nécessairement le plus proche de chez eux. Ils privilégient les qualités spécifiques des espaces (taille, possibilité d'avoir un bureau fermé ou de stocker du matériel) et surtout le réseau social et amical qui s'y trouve, comme l'explique Rosa, traductrice :

[Ici], j’ai bien accroché avec l'équipe quand j'ai visité. Et puis, au niveau de l'accès... En fait, en kilomètres, je pense que ce n'est pas beaucoup moins loin que M., mais au niveau de l'accès, c'est direct depuis chez moi. Je mets vingt minutes, sans ralentissement. [...] [S'il y avait un espace de coworking qui ouvrait dans ma commune], je ne suis pas sûre que ça m'intéresserait. Parce qu'ici, je me sens bien aussi. [...] Par exemple, il est question d'en ouvrir un à L., qui est juste à côté de V., à dix minutes, qui serait plus proche. Et dans la mesure où je me sens bien ici, je n'irai pas à L. parce que c'est plus proche. 
La plupart des coworkers sont bien conscients que le travail dans un EC génère pour eux des déplacements supplémentaires par rapport au travail à domicile. Mais, alors que c'est l'habitat en maison individuelle qui est généralement plébiscité et qui implique le plus souvent d'être éloigné du centre-ville (Charmes, 2019), les EC permettent d'avoir accès aux aménités urbaines : toujours situés dans les centres, ils sont proches des commerces, écoles et services et sont donc vecteurs d'une proximité retrouvée avec les centres-villes en milieu rural. Comme l'explique Paul, chargé de projets informatiques en Ardèche :

Parce que c'est central, parce que l'idée justement, c'est de sortir de chez soi et d'avoir ce petit côté un peu urbain aussi en ce qui me concerne. Ce petit côté, je vais au boulot, je vais en ville et puis le soir quand je sors, il y a la boulangerie etc. [...] C'est franchement parce qu'on a envie de travailler dans le centre-ville parce qu'en termes de transports, c'est sûr que c'est pas le plus pratique en fait, tu te retrouves à bosser à la campagne à douze kilomètres de chez toi et moi ça me prend, franchement, porte à porte ça me prend au moins vingt-cinq minutes.

On constate que la limite de définition de la notion de proximité varie d'un coworker à l'autre : pour certains, elle se définit par une accessibilité à pied et le fait de vivre et travailler dans la même commune, tandis que pour d'autres le déplacement est une occasion attendue de quitter le hameau ou le village pour le bourg d'à côté. Dans tous les cas, la liberté de choisir soi-même la limite au-delà de laquelle on ne consent plus au déplacement distingue nettement les coworkers du modèle de "navetteur " qu'ils veulent à tout prix éviter, comme l'explique Julien, développeur informaticien installé en Isère :

Oui, pour moi [l'espace F.] c'est quelque chose près de moi, parce qu'en fait je trouve que, si j'habite là, c'est pas pour descendre tous les jours à Grenoble, avec les bouchons, je connais plein de gens qui descendent tous les jours parce qu'ils sont obligés, ils ont déjà travaillé avant à Grenoble, après ils ont déménagé par ici, ils ont acheté une maison, ils ont construit, parfois c'est super compliqué en fait. Et voilà pour moi la priorité c'était pas aller plus loin que le quart d'heure en voiture. 
On constate donc que pour les coworkers, la proximité correspond avant tout à une distance choisie et susceptible de varier d'un individu à l'autre, notamment en fonction des modes de transport utilisés, puisqu'il s'agit avant tout d'une limite temporelle (de l'ordre de vingt minutes environ), qui participe à la ritualisation de la coupure entre vie privée et vie professionnelle (Flipo \& Ortar, 2020).

Des trajets domicile-travail qui demeurent dominés par l'automobile, mais une surreprésentation des modes doux

Les résultats de l'étude quantitative montrent tout d'abord un taux d'équipement élevé en moyens de transports individuels. L’ensemble des ménages interrogés dispose d'au moins un véhicule à moteur (voiture, scooter ou moto), $88 \%$ en possèdent au moins deux ${ }^{9}$, et $56 \%$ en ont même trois et plus. Ce taux élevé reflète l'importance des ménages bi-actifs et l'appartenance aux catégories sociales supérieures des coworkers. $37 \%$ de ces derniers vivent et travaillent dans la même commune, ce qui est à peu près conforme à la moyenne en AuvergneRhône-Alpes (Coudène et Lévy, 2017). Cependant, la distance moyenne s'élève à 7,3 kilomètres : c'est moitié moins que la moyenne des actifs en milieu rural (ENTD, 2008), bien qu'on observe une hétérogénéité de situations avec des distances allant jusqu'à une trentaine de kilomètres.

\begin{tabular}{|c|c|}
\hline Même commune & $37 \%$ \\
\hline Jusqu'à $5 \mathrm{~km}$. & $14 \%$ \\
\hline De 6 à $10 \mathrm{~km}$. & $16 \%$ \\
\hline $11 \mathrm{~km}$ et plus & $33 \%$ \\
\hline
\end{tabular}

Tableau 1 - Distance entre le domicile et l'EC.

Source : exploitation qualitative des données de l'enquête quantitative, calcul sur la base des distances routières entre communes de résidence.

9 La moyenne départementale des ménages bimotorisés s'élève à $41 \%$ pour la Drôme et $44 \%$ pour l'Ardèche. 
Cette moindre amplitude kilométrique moyenne peut expliquer la moindre utilisation de la voiture, qui demeure néanmoins dominante : $53 \%$ des coworkers l'utilisent comme principal mode de transport pour se rendre dans l'EC en ruralité, contre seulement $2 \%$ dans les centresvilles des grandes agglomérations, mais contre $70 \%$ dans le périurbain. L'utilisation de l'automobile croît avec la distance, mais son utilisation demeure moindre que la moyenne : si un quart des coworkers qui vivent et travaillent dans la même commune l'utilisent, c'est le cas de $51 \%$ de l'ensemble des actifs dans le même cas (Coudène \& Lévy, 2016). Cependant, l'utilisation de la voiture comme mode de transport exclusif est généralisé au-delà de onze kilomètres, et un seul enquêté a déclaré utiliser le covoiturage en complément.

Le vélo arrive en second rang : d'après notre enquête, $17 \%$ des coworkers l'utilisent en milieu rural, contre $26 \%$ en ville. C'est bien davantage que la moyenne régionale qui s'élève à $2,2 \%$, et même que la moyenne observée en milieu urbain : à Lyon, $8 \%$ des actifs se rendent au travail à vélo ; à Grenoble, qui est l'une des villes de France où le vélo est le plus répandu, ce taux s'élève à $15 \%$ (Tallet \& Vallet, 2017). Les deux tiers des utilisateurs du vélo vivent et travaillent dans la même commune, mais on trouve également quelques cyclistes qui vont jusqu'à dix kilomètres.

Enfin, la marche est le troisième mode le plus emprunté : $10 \%$ des coworkers en milieu rural l'utilisent, ce qui est beaucoup moins que les coworkers urbains (25\%). De manière assez attendue, aucun coworker en milieu rural n'utilise les transports en commun pour se rendre à l'EC.

D’une manière générale, on constate une grande omnivorité des modes de transport utilisés, en particulier eu égard à l'environnement considéré : au-delà des trajets entre le domicile et l'EC, $20 \%$ des coworkers utilisent régulièrement le train, $18 \%$ les transports en commun - ce qui indique la fréquentation régulière d'une grande ville -, $28 \%$ le covoiturage. En outre, 19 \% d'entre eux déclarent pratiquer la marche quotidiennement. Ainsi, bien que la voiture domine, les modes doux sont surreprésentés. 


\section{La surreprésentation des modes doux : entre effet de sélection et diffusion}

Cette surreprésentation s'explique d'abord par un effet de sélection : la population jeune et très diplômée qui fréquente les EC est souvent sensibilisée aux enjeux écologiques, et nombreux sont les tiers-lieux qui ambitionnent d'être des « laboratoires du changement social " (BerrebiHoffman, Bureau \& Lallement, 2018) vers « des modes de vie durables ${ }^{10} »$. On trouve ainsi fréquemment dans les tiers-lieux des formes d'économie collaborative, circulaire ou participative : paniers $\mathrm{AMAP}^{11}$, achats groupés, covoiturage, zones de gratuité, etc.

Cette affinité peut aussi s'expliquer par la proximité sociale entre différents groupes d'acteurs porteurs de ces innovations (Berrebi-Hoffman, Bureau \& Lallement, 2018). Les individus réunis dans les EC, bien que distincts par leurs secteurs d'activité, se retrouvent sur un ensemble de préoccupations environnementales et sociales ainsi que sur une socialisation commune, notamment par la fréquentation des études supérieures et donc des grandes villes. Or, c'est bien souvent via l'expérience urbaine que la socialisation aux « modes doux » s'opère ${ }^{12}$.

Par ailleurs, une partie des coworkers a anticipé le fait que leur relocalisation à la campagne allait engendrer une dépendance plus forte à l'égard de l'automobile. Si cette dépendance est jugée inévitable, comme l'indique le fait que les nouveaux arrivants ont tous fait l'acquisition d'une voiture et, pour certains, ont passé un permis de conduire dont ils n'avaient jusque-là jamais eu besoin, elle est toutefois vécue comme un inconvénient à limiter. Les trajets domicile-travail sont le pivot de cette stratégie : vivre et travailler dans la même commune - que ce soit en choisissant de vivre à proximité d'un $\mathrm{EC}$, d'en rejoindre un proche de chez soi, voire d'en créer un, - participe à l'évitement de la voiture dans les trajets quotidiens. La voiture est alors principalement dévolue aux

\footnotetext{
10 Telle est la formulation utilisée par le collectif Movilab qui porte le mouvement des tiers-lieux français.

11 Association pour le Maintien d'une Agriculture Paysanne.

12 Cf. la communication de l'auteure, "Becoming a Rural Cyclist ", lors du Worshop Becoming Urban Cyclists: From Socialization to Skills, organisé par le Laboratoire Aménagement Économie Transports (LAET) de l'École de l'aménagement durable des territoires (ENTPE), à Lyon, du 10 au 11 février 2020.
} 
motifs de loisirs, aux sorties en famille et à l'accès aux aménités environnementales qui ont motivé, dans de nombreux cas, l'installation dans la région. On observe ici une tendance clairement inverse à celle qui a pu être observée au sujet des ménages populaires, dans lesquels l'usage de la voiture pour des motifs de loisirs est réduit au profit des trajets contraints (Hubert, Pistre \& Madre, 2016).

De même, on constate une partition nette entre les coworkers qui, bien quayant fréquenté la ville, ont grandi en milieu rural ou périurbain et pour qui la voiture n'est pas perçue comme une contrainte, et les citadins "de naissance " pour qui la voiture est perçue comme l'inconvénient par excellence de la campagne. Ainsi pour Simon, " en tant que citadin, j'ai trop vu les limites de la voiture et les nuisances qu'elles génèrent ", alors que pour Aymeric, " ayant grandi entre Lyon et Saint-Étienne, j’ai l'habitude de faire beaucoup de voiture, ça ne me dérange pas ». Ces différences révèlent l'intrication des socialisations primaires et secondaires en matière de mobilité.

Bien que minoritaire, la forte valorisation dont bénéficient les modes doux dans les EC peut contribuer à leur diffusion, par la socialisation aux pratiques durables et par l'échange d'informations. Par ailleurs, y compris chez les coworkers qui n'expriment pas de sensibilité écologique, la relocalisation dans les campagnes s'accompagne souvent d'une volonté de mieux maîtriser sa mobilité. L'idéal du village s'accompagne d'un désir de " poser ses valises ", comme l'expliquent plusieurs coworkers qui ont été de grands mobiles à l'international : "j'ai beaucoup voyagé, j'étais toujours entre deux avions [...] j'ai eu envie de poser mes valises » explique Maëlle, photographe ayant vécu dix ans au Moyen-Orient. "Moi j'avais passé dix ans, quand je dis Maroc, c'est Maroc avec beaucoup de voyages allers-retours, Europe, avion... ", explique également Stéphane, consultant informatique, qui dit avoir voulu « changer de rythme » en s'installant à la campagne. Dans ce processus, l'EC apparaît comme un outil central de la reconstruction d'un système de proximité en milieu rural, qui permet aux coworkers de conserver une activité fortement qualifiée, tout en n'ayant pas à subir de longs déplacements jusqu'aux grandes villes. Si elle n'est pas nécessairement recherchée pour ses vertus écologiques, la mobilité douce peut être privilégiée pour la qualité de vie. 


\section{Conclusion}

En conclusion, on constate que les tiers-lieux ont des effets mitigés sur les mobilités en milieu rural. Ils contribuent à la fois à une augmentation générale de la mobilité, qu'elle soit quotidienne ou résidentielle, et à une diffusion des modes de vie des hypercentres urbains - y compris par la valorisation de la proximité et de l'usage des modes doux - dans les campagnes. Si les mobilités quotidiennes y sont plus vertueuses sur le plan écologique que la moyenne des mobilités domicile-travail, les EC créent néanmoins une demande de mobilité nouvelle et génèrent mécaniquement de nouveaux déplacements, plus qu'ils n'en suppriment. Ainsi, parmi les personnes que nous avons rencontrées et interrogées, nous n'avons trouvé aucun profil de télétravailleur " navetteur ", c'est-à-dire pour qui le travail dans un EC se substitue effectivement à un déplacement quotidien. En revanche, en facilitant les transitions professionnelles et résidentielles, les EC participent effectivement de l'attractivité résidentielle des territoires - bien que leur implantation démontre qu'ils en sont davantage le résultat que l'origine.

Enfin, il faut noter que parmi les nouveaux déplacements générés par ces espaces, certains contribuent à recréer de nouvelles zones de chalandise, dont certains villages et petites villes avaient été entièrement dépourvus. Étant donné qu'ils sont toujours situés dans les centresbourgs, proches des services et des commerces, les EC ne sont pas promoteurs d'une mobilité entièrement motorisée, telle que celle qui est promue par les zones d'activités aménagées en dehors des villes. En ce sens, ils contribuent à recréer des espaces de proximité plus favorables aux modes doux. La présence de militants de la mobilité douce, en particulier cyclistes, contribue par ailleurs à faire pression sur les pouvoirs publics locaux pour améliorer les équipements dont d'autres habitants peuvent bénéficier.

Si les EC peuvent contribuer à promouvoir la transition mobilitaire, ce n'est donc pas quantitativement par une réduction des déplacements, mais plutôt par le biais de la diffusion de pratiques et de modes de vies plus axés sur la proximité. Ceux-ci ne sont toutefois pas accessibles à tous, et les EC se font alors le reflet de nouvelles inégalités de mobilité, 
non pas fondées sur l'accès à la mobilité mais sur le contrôle et la réduction des mobilités contraintes, intrinsèquement liée au contrôle des temporalités quotidiennes.

\section{Références bibliographiques}

Agence de l'Environnement et de la Maîtrise de l'Énergie (ADEME), 2014. Alléger l'empreinte environnementale de la consommation des Français en 2030. Vers une évolution profonde des modes de production et de consommation, Rapport de synthèse [En ligne]. Mis en ligne en 11/2014 (consulté le 25/11/2020). URL : https://www.ademe.fr/alleger-lempreinteenvironnementale-consommation-francais-2030-synthese

Aguilera Anne, Lethiais Virginie, Rallet Alain \& Proulhac Laurent, 2016. "Home-Based Telework in France: Characteristics, Barriers and Perspectives ", Transportation Research Part A: Policy and Practice [En ligne], 92, p. 1-11. Mis en ligne le 26/07/2016 (consulté le 27/11/2020). URL : https://www.sciencedirect.com/science/article/pii/S0965856416305742 ; DOI : 10.1016/j.tra.2016.06.021

Bentayou Gilles, Hasiak Sophie, Marrel Joris, Perrin Emmanuel \& Richer Cyprien, 2019. Les espaces de coworking : des outils au service de la mobilité durable ?, Rapport final [En ligne], Centre d'études et d'expertise sur les risques, l'environnement, la mobilité et l'aménagement (CEREMA). Mis en ligne le 18/10/2019 (consulté le 27/11/2020). URL : https://www.cerema.fr/ fr/actualites/espaces-coworking-outils-au-service-mobilite-durable

Berrebi-Hoffmann Isabelle, Bureau Marie-Christine \& Lallement Michel, 2018. Makers. Enquête sur les laboratoires du changement social, Paris, Éditions du Seuil.

Besson Raphaël, 2018. «Les tiers-lieux : des outils de régénération économique des territoires ruraux ? », in M. Talandier \& B. Pecqueur (dir.), Renouveler la géographie économique, Paris, Economica, p. 121-136.

Boboc Anca, Bouchareb Kevin, Deruelle Valérie \& Metzger JeanLuc, 2014. "Le coworking : un dispositif pour sortir de l'isolement?", SociologieS, [En ligne]. Mis en ligne le 10/11/2014 (consulté le 03/05/2018), URL : http://journals.openedition.org/sociologies/4873

Burret Antoine, 2017. Étude de la configuration en Tiers-Lieu. La repolitisation par le service, thèse de doctorat, sous la dir. de G. Herreros, Université Lyon II. 
Caisse des Dépôts, 2014. Rapport d'étude sur les externalités des télécentres.

Commissariat Général à l'Égalité des Territoires (CGET), 2015. Étude sur les opportunités des territoires à développer le télétravail, mobilisation des gains pour les individus, les entreprises et les territoires.

Charmes Éric, 2019. La revanche des villages. Essai sur la France périurbaine, Paris, Éditions du Seuil.

Claisse Gérard, 1997. L'abbaye des télémythes : techniques, communication et société, Lyon, Aléas.

Coudène Maud \& Levy David, 2016. «De plus en plus de personnes travaillent en dehors de leur commune de résidence ", Insee Première [En ligne], 1605. Mis en ligne le 30/06/2016 (consulté le 27/11/2020). URL : https://www. insee.fr/fr/statistiques/2019022

FLIPo Aurore, 2020. "Espaces de coworking et tiers-lieux. Les réseaux d'une nouvelle ruralité ?", Études rurales [En ligne], 206, p. 154-174. Mis en ligne le 01/01/2021 (consulté le 08/02/2021). URL : http://journals.openedition. org/etudesrurales/23887 ; DOI : 10.4000/etudesrurales.23887

Flipo Aurore \& Lejoux Patricia, 2020. «Les dimensions sociales et spatiales du coworking. Un état de l'art ", EspacesTemps.net [En ligne]. Mis en ligne le 03/04/2020 (consulté le 26/11/2020) URL : https://www.espacestemps.net/ articles/les-dimensions-sociales-et-spatiales-du-coworking-un-etat-de-lart/

Flipo Aurore \& Ortar Nathalie, 2020. "Séparer les espaces pour maîtriser le temps. La reconstruction des barrières temporelles et spatiales entre vie privée et vie professionnelle par le coworking", Temporalités [En ligne], 31-32. Mis en ligne le 03/02/2021 (consulté le 08/02/2021). URL : http://journals. openedition.org/temporalites/7712 ; DOI : 10.4000/temporalites.7712

Gandini Alessandro, 2015. "The Rise of Coworking Spaces: A Literature Review ", Ephemera: Theory and Politics in Organization [En ligne], 15 (1), p. 193-205. Consulté le 26/11/2020. URL : http://www.ephemerajournal. org/contribution/rise-coworking-spaces-literature-review

Genoud Patrick \& Moeckli Alexis, 2010. «Les tiers-lieux, espaces d'émergence et de créativité ", Revue économique et sociale, 68 (2), p. 25-33.

Gill Rosalind \& Pratt Andy, 2008. "In the Social Factory?: Immaterial Labour, Precariousness and Cultural Work ", Theory, Culture \& Society [En ligne], 25 (7-8), p. 1-30. Mis en ligne le 01/12/2008 (consulté le 26/11/2020). URL : https://journals.sagepub.com/doi/10.1177/0263276408097794 ; DOI : $10.1177 / 0263276408097794$ 
Hubert Jean-Paul, Pistre Pierre \& Madre Jean-Loup, 2016. «L'utilisation de l'automobile par les ménages dans les territoires peu denses. Analyse croisée par les enquêtes sur la mobilité et le Recensement de la population ", Économie et Statistique [En ligne], 483-484-485, p. 179-203. Mis en ligne le 28/04/2016 (consulté le 27/11/2020). URL : https://www.insee.fr/fr/ statistiques $/ 2017650$ ?sommaire $=2017660$

Leducq Divya, Demazière Christophe \& Coquel Aurélie, 2019. «Diffusion régionale et intégration urbaine des espaces de coworking. Les spécificités d'une région française faiblement métropolisée ", Géographie, économie, société [En ligne], 21 (3), p. 145-169. Mis en ligne le 16/10/2019 (consulté le 26/11/2020). URL : https://www.cairn-int.info/revue-geographieeconomie-societe-2019-3-page-145.htm

Lejoux Patricia, Flipo Aurore, Ortar Nathalie, Ovtracht Nicolas, SoucheLecorvec Stéphanie \& Stanica Razvan, 2019. "Coworking, a Way to Achieve Sustainable Mobility? Designing an Interdisciplinary Research Project ", Sustainability, 11 (24), 7161.

Levy-Waitz Patrick, 2018. Faire ensemble pour mieux vivre ensemble. Rapport de la mission "Coworking travail et numérique ", Fondation travailler autrement [En ligne], Commissariat général de l'égalité des territoires (CGET), ministère de la Cohésion des territoires. Mis en ligne le 29/09/2018 (consulté le 25/11/2020). URL : https://www.fondation-travaillerautrement.org/2018/09/19/mission-coworking-faire-ensemble-pour-mieuxvivre-ensemble/

Moeckel Rolf, 2017. "Working from Home: Modeling the Impact of Telework on Transportation and Land Use ", Transportation Research Procedia [En ligne], 26, p .207-214. Mis en ligne le 14/08/2017 (consulté le 27/11/2020). URL : https://www.sciencedirect.com/science/article/pii/ S2352146517308803 ; DOI : 10.1016/j.trpro.2017.07.021

Moriset Bruno, 2004. "Télétravail, travail nomade : le territoire et les territorialités face aux nouvelles flexibilités spatio-temporelles du travail et de la production ", Cybergeo: European Journal of Geography, [En ligne], document 257. Mis en ligne le 06/02/2004 (consulté le 02/07/2018). URL : https://journals.openedition.org/cybergeo/3815 ; DOI : 10.4000/ cybergeo.3815

Oldenburg Ray, 1999 [1989]. The Great Good Place: Cafés, Coffee Shops, Bookstores, Bars, Hair Salons, and Other Hangouts at the Heart of a Community, Cambridge (Mass.), Da Capo Press. 
Ortar Nathalie, 2009. "Entre choix de vie et gestion des contraintes. Télétravailler à la campagne », Flux [En ligne], 78 (4), p. 49-57. Mis en ligne le 01/01/2010 (consulté le 26/11/2020). URL : https://www.cairn.info/ revue-flux1-2009-4-page-49.htm ; DOI : 10.3917/flux.078.0049

Pistre Pierre, 2012. Renouveaux des campagnes françaises. Évolutions démographiques, dynamiques spatiales et recompositions sociales, thèse de doctorat, sous la dir. de C. Rhein, Université Paris Diderot.

Recchi Ettore, Flipo Aurore \& Duwez Emmanuelle, à paraître en mars 2021. "Ce monde que je connais : les space-sets des Français », in P. Mercklé \& E. Duwez, Un panel français. L'étude longitudinale par Internet pour les sciences sociales (ELIPSS), Paris, Éditions de l'INED.

Salgueiro Louis, 2015. Les dynamiques territoriales d'adoption, de diffusion et d'usages des tiers-lieux de travail ruraux. Une approche systémique des télécentres du Cantal, thèse de doctorat, sous la dir. de G. Puel \& V. Fernandez, Université Toulouse 2.

Scaillerez Arnaud \& Tremblay Diane-Gabrielle, 2017. "Coworking, fab labs et living labs. État des connaissances sur les tiers lieux ", Territoire en mouvement. Revue de géographie et aménagement [En ligne], 34, Mis en ligne le 16/06/2017 (consulté le 28/05/2018). URL : https://journals.openedition. org/tem/4200 ; DOI : 10.4000/tem.4200

SpINuzzi Clay, 2012. "Working Alone Together: Coworking as Emergent Collaborative Activity", Journal of Business and Technical Communication [En ligne], 26 (4), p. 399-441. Mis en lignele 30/05/2012 (consultéle 26/11/2020). URL : https://journals.sagepub.com/doi/abs/10.1177/1050651912444070 ; DOI $: 10.1177 / 1050651912444070$

TAllet Frédéric \& VAllet Vincent, 2017. «Partir de bon matin, à bicyclette... », Insee Première [En ligne], 1629. Mis en ligne le 17/01/2017 (consulté le 27/11/2020). URL : https://www.insee.fr/fr/statistiques/2557426

Tinetti Benoit, Veron Sarah de, Duvernois Pierre-Alexis, Brun Olivier, Roucher Clément, Olagne Régis \& Chamignon Delphine, 2015. Évaluation de l'impact du télétravail \& des tiers lieux sur la réduction des consommations d'énergie et émissions de gaz à effet de serre, et sur l'organisation des entreprises, Rapport final [En ligne], ADEME. Mis en ligne en 09/2020 (consulté le 26/11/2020). URL : https://www.ademe.fr/evaluation-limpactteletravail-tiers-lieux-reduction-consommations-denergie-emissions-gaz-aeffet-serre-lorganisation 
Tissandier Patrice \& Mariani-Rousset Sophie, 2019. " Les bénéfices du télétravail. Mobilité modérée : réduction du stress et des émissions de gaz à effets de serre ", Revue francophone sur la santé et les territoires [En ligne]. Mis en ligne le 27/05/2019 (consulté le 08/01/2020). URL : rfst.hypotheses.org/ tissandier-patrice-mariani-rousset-sophie

Torre André, 2018. "Développement territorial et relations de proximité ", Revue d'Économie Régionale \& Urbaine [En ligne], 5, p. 1043-1075. Mis en ligne le 10/01/2019 (consulté le 26/11/2020). URL : https://www.cairn. info/revue-d-economie-regionale-et-urbaine-2018-5-page-1043.htm ; DOI : $10.3917 /$ reru. 185.1043 



\section{PEUT-ON SE PASSER DE LA VOITURE HORS DES CENTRES URBAINS ?}

S'inscrivant dans le cadre du projet Construire des mobilités durables, inclusives et responsables (CONDUIRE), financé par l'Agence De l'Environnement et de la Maîtrise de l'Énergie (ADEME), les actes du colloque "Peut-on se passer de la voiture hors des centres urbains ? », soutenu par la MSH Paris-Saclay, cherchent à interroger les pratiques de déplacements des ménages en zones peu denses. Cet ouvrage a ainsi pour ambition de comprendre les ressorts de la transition vers des mobilités plus durables, au sein des espaces où l'automobile reste prégnante.

Le fil rouge de l'ouvrage est le paradoxe selon lequel les solutions de substitution à la voiture sont proposées et mises en place quasi exclusivement dans les espaces denses, pourtant les moins dépendants à l'automobile. Les espaces périphériques, pour autant caractérisés par un usage intensif et croissant de la voiture, connaissent peu d'alternatives écologiques et inclusives à l'automobile. Or, le développement de la transition économique, écologique et sociale ne peut faire l'économie d'une étude approfondie des usages de la voiture dans de tels espaces. D'une part, ces espaces contribuent d'une façon importante à la pollution atmosphérique ; d'autre part, ils concentrent les populations pour lesquelles la voiture est à la fois indispensable, contraignante et coûteuse, comme le mouvement social des Gilets jaunes l'a laissé entrevoir.

L'ouvrage cherche à interroger la dépendance automobile à plusieurs échelles temporelles et spatiales, tout en proposant une analyse des alternatives à la voiture au sein des zones les moins denses. Les quatre premiers chapitres proposent un panorama historique et spatial de la dépendance automobile, amenant des réflexions sur le futur de la voiture dans ces espaces. Dans un deuxième temps, l'ouvrage interroge les représentations et les usages contemporains de l'automobile hors des villes. La troisième partie s'attache enfin à évoquer les solutions alternatives à l'automobile au sein de ces espaces : tiers-lieux, covoiturages et motorisation électrique.
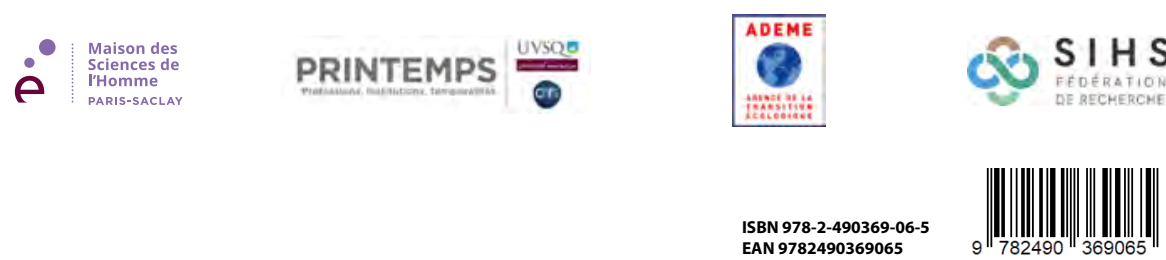